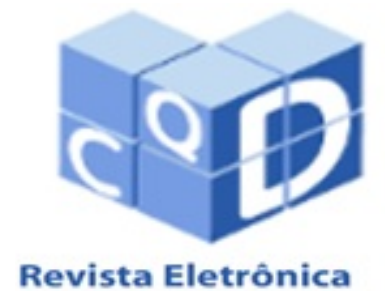

Revista Eletrônica
Paulista de Matemática

ISSN 2316-9664

Volume 13, dez. 2018

Raphael de Oliveira Garcia

Universidade Federal de São

Paulo

gr.gubim@gmail.com

Graciele Paraguaia Silveira

Universidade Federal de São

Carlos

gracimat@gmail.com

\section{Soluções numéricas de EDO's aplicadas no estudo de dinâmica populacional}

Numerical solutions of ODEs applied in the study of population dynamics

\section{Resumo}

O estudo de EDO's que modelam dinâmicas populacionais é cada vez mais importante pois permite prever comportamentos e auxiliar na tomada de decisões sobre aspectos relevantes da sociedade como epidemiologia, controle biológico, demografia, entre outros. Este trabalho teve como propósito a análise de métodos numéricos para EDO's, aplicadas em problemas clássicos de dinâmica populacional. O objetivo foi comparar métodos e estudos numéricos de suas respectivas ordens de convergência. Os métodos de passo simples considerados foram Euler, Euler Modificado, Ponto Médio e Runge-Kutta Clássico. Já os métodos de múltiplos passos foram Adams-Bashforth de 2 e 4 pontos e Adams-Moulton de 2 e 4 pontos. Os códigos próprios foram escritos no Octave. Os resultados mostraram que nem sempre métodos explícitos de maior ordem de convergência, de passo simples, proporcionarão soluções numéricas consideradas mais adequadas.

Palavras-chave: Equações diferenciais ordinárias. Métodos numéricos. Dinâmica populacional. Ordem de convergência.

\begin{abstract}
The study of ODEs that model population dynamics is very important because allows to predict behavior and assist decision making about relevant aspects of the society such as epidemiology, biological control, demography, among others. The purpose of this study was to analyse numerical methods for ODEs, applied in classic problems of population dynamics. The aim was to compare methods and numerical studies of their respective convergence order. The one-step methods considered were Euler, Modified Euler, MidPoint and classic Runge-Kutta. The multiplestep methods were Adams-Bashforth 2 and 4 points and AdamsMoulton 2 and 4 points. The own codes were written on Octave. The results showed that not always explicit methods of greater order of convergence, of simple step, will provide numerical solutions considered more adequate.
\end{abstract}

Keywords: Ordinary differential equations. Numerical methods. Populational dynamics. Convergence order. 


\section{Introdução}

O uso da modelagem matemática para descrever fenômenos reais vem acompanhando a humanidade desde a Idade Antiga até a atualidade. Em diversas situações, a dificuldade e até mesmo a impossibilidade de se obter soluções analíticas de equações, oriundas do processo de modelagem, fizeram com que métodos numéricos e computacionais ganhassem cada vez mais espaço, na busca por soluções aproximadas em aplicações nas Ciências como um todo.

O desenvolvimento de técnicas numéricas e computacionais gerou múltiplas opções de esquemas, modelagens e linguagens, hoje disponíveis para se trabalhar com modelagem matemática. Neste sentido, estudos e pesquisas vêm sendo feitos com o intuito de realizar comparações entre modelos matemáticos, métodos numéricos e linguagens [1, 2, 3, 4, 5].

$\mathrm{Na}$ área de equações diferenciais existem variadas opções de métodos numéricos, todavia, um uso ingênuo de tais métodos pode fazer com que conclusões equivocadas sejam produzidas. Por exemplo, resultados que induzam a se levar em consideração soluções que, aparentemente representam a solução analítica, mas que na verdade não condizem com o fenômeno modelado pela equação diferencial, deixando portanto de ser uma aproximação adequada para a solução exata.

Equações diferenciais ordinárias que modelam dinâmicas populacionais são de grande interesse nas ciências, pois a partir delas é possível prever comportamentos que possam auxiliar em tomadas de decisões, desde decisões associadas a epidemiologia, controle biológico, demografia, entre outros. Assim, neste ambiente uma solução numérica com erros espúrios pode afetar drasticamente uma tomada de decisão relacionada a algum problema real.

Neste trabalho considerou-se métodos numéricos de equações diferencias ordinárias aplicadas em problemas clássicos de dinâmica populacional, com o objetivo de realizar comparações entre os métodos e estudos numéricos de suas respectivas ordens de convergência. Os métodos de passo simples explícitos considerados foram Método de Euler, Método de Euler Modificado, Método do Ponto Médio ou Método Midpoint e o Método de Runge-Kutta Clássico. Os métodos de múltiplos passos explícitos foram Adams-Bashforth de 2 e de 4 pontos e os métodos de múltiplos passos preditor-corretor foram Adams-Moulton de 2 e de 4 pontos $[6,7,8]$. Toda a implementação computacional foi feita no Octave, versão 4.2.1 [9], sem o uso de pacotes prontos para as implementações, isto é, com códigos próprios.

Na Seção 2 estão descritos os métodos numéricos utilizados e o procedimento adotado para efetuar o estudo numérico da ordem de convergência dos mesmos. Na Seção 3 encontram-se os problemas de dinâmica populacional nos quais os métodos numéricos foram empregados e por fim, na Seção 4 tem-se as conclusões do trabalho.

\section{Métodos numéricos}

Nesta seção estão descritos os métodos numéricos escolhidos para se obter soluções aproximadas das equações diferenciais clássicas, que modelam dinâmica populacional. Para isso, sem perda de generalidade, consideramos um Problema de Valor Inicial (PVI), conhecido como problema de Cauchy, definido por: 


$$
\left\{\begin{array}{l}
\frac{d x}{d t}=f(t, x), \quad t \in I \subset \mathbb{R} \\
x(0)=x_{0}
\end{array},\right.
$$

em que $f: I \times \mathbb{R} \rightarrow \mathbb{R}$ é uma função conhecida e $x=x(t), x: I \rightarrow \mathbb{R}$, é a função a ser encontrada [7].

Inicialmente serão apresentados os esquemas de passo simples escolhidos, a saber, método de Euler, método de Euler modificado, método do Ponto Médio ou método Midpoint e o método de Runge-Kutta clássico [6, 7]. Para todos os esquemas numéricos realizou-se a seguinte discretização.

Considere um PVI conforme a expressão (1), definido para $t \in\left[0, t_{f}\right] \subset \mathbb{R}$. Seja

$$
\Pi: 0=t_{0}<t_{1}<\ldots<t_{N}=t_{f}
$$

uma partição regular de $\left[0, t_{f}\right] \operatorname{com} N$ subintervalos e espaçamento $h=\left(t_{f}-t_{0}\right) / N$. Quando for possível, denota-se a solução exata em um ponto $t=t_{i}, i=0, \ldots, N$, por $x_{i}=x\left(t_{i}\right)$ e por $\xi_{i}$ a solução aproximada fornecida por algum esquema numérico específico. Assim, o que difere um esquema do outro é a forma pela qual $\xi_{i+1}$ está definida e o conjunto $\left\{\xi_{0}=x_{0}, \xi_{1}, \ldots, \xi_{N}\right\}$ é uma solução aproximada do PVI (1).

\subsection{Método de Euler}

No esquema de Euler explícito de primeira ordem, a solução aproximada $\xi_{i+1}$ é definida por

$$
\xi_{i+1}=\xi_{i}+h f\left(t_{i}, \xi_{i}\right), \quad i=0, \ldots, N-1 .
$$

O intervalo de estabilidade absoluta $J$ de um método numérico pode ser obtido ao aplicá-lo em um PVI (1), cuja função $f$ é definida por $f(t, x)=\lambda x(t)$, com $\lambda<0$. Se a solução numérica encontrada satisfaz a condição $\lim \xi_{n}=0$ para $\lambda h \in J$, então $J$ é o intervalo de estabilidade do método em questão. Para o método de Euler tem-se $J=(-2,0)$ [6].

\subsection{Método de Euler modificado}

No esquema de Euler modificado explícito de segunda ordem, a solução aproximada $\xi_{i+1}$ é definida por

$$
\left\{\begin{array}{l}
m_{1}=f\left(t_{i}, \xi_{i}\right) \\
m_{2}=f\left(t_{i}+h, \xi_{i}+h m_{1}\right) \\
\xi_{i+1}=\xi_{i}+\frac{h}{2}\left[m_{1}+m_{2}\right]
\end{array}, \quad i=0, \ldots, N-1,\right.
$$

em que o intervalo de estabilidade absoluta é dado por $J=(-2,0)$ [6]. 


\subsection{Método do Ponto Médio}

Para o método do Ponto Médio de segunda ordem, também chamado de método Midpoint, a solução aproximada $\xi_{i+1}$ é definida por

$$
\left\{\begin{array}{l}
m_{1}=f\left(t_{i}, \xi_{i}\right) \\
m_{2}=f\left(t_{i}+\frac{h}{2}, \xi_{i}+\frac{h}{2} m_{1}\right), \quad i=0, \ldots, N-1 . \\
\xi_{i+1}=\xi_{i}+h m_{2}
\end{array}\right.
$$

Esse método é instável [6], isto é, não existe um intervalo $J$ que possa garantir que $\lim \xi_{n}=0$ para $\lambda \in J$.

\subsection{Método de Runge-Kutta clássico}

No método de Runge-Kutta de quarta ordem, a solução aproximada $\xi_{i+1}$ é definida por

$$
\left\{\begin{array}{l}
m_{1}=f\left(t_{i}, \xi_{i}\right) \\
m_{2}=f\left(t_{i}+\frac{h}{2}, \xi_{i}+\frac{h}{2} m_{1}\right) \\
m_{3}=f\left(t_{i}+\frac{h}{2}, \xi_{i}+\frac{h}{2} m_{2}\right) \\
m_{4}=f\left(t_{i}+h, \xi_{i}+h m_{3}\right) \\
\xi_{i+1}=\xi_{i}+\frac{h}{6}\left[m_{1}+2 m_{2}+2 m_{3}+m_{4}\right]
\end{array}, \quad i=0, \ldots, N-1 .\right.
$$

Neste caso, o intervalo de estabilidade absoluta é dado por $J=(-2,78,0)$ [6].

A seguir tem-se os métodos explícitos de múltiplos passos, escolhidos para abordagem neste estudo.

\subsection{Método de Adams-Bashforth 2}

No esquema explícito de Adams-Bashforth, de dois pontos e de segunda ordem, a solução aproximada $\xi_{i+1}$ é definida por

$$
\xi_{i+1}=\xi_{i}+\frac{h}{2}\left[3 f\left(t_{i}, \xi_{i}\right)-f\left(t_{i-1}, \xi_{i-1}\right)\right], i=1, \ldots, N-1 .
$$

Note que $\xi_{1}$ não está definida na equação (6), fazendo com que $\xi_{1}$ tenha que ser calculada por um esquema de passo simples. Neste trabalho utilizou-se o método de Euler modificado definido pela equação (3). O intervalo de estabilidade absoluta é dado por $J=(-1,0)$ [6].

\subsection{Método de Adams-Bashforth 4}

Para o esquema de Adams-Bashforth explícito, de quatro pontos e de quarta ordem, a solução aproximada $\xi_{i+1}$ é definida por

$$
\xi_{i+1}=\xi_{i}+\frac{h}{24}\left[55 f\left(t_{i}, \xi_{i}\right)-59 f\left(t_{i-1}, \xi_{i-1}\right)+37 f\left(t_{i-2}, \xi_{i-2}\right)-9 f\left(t_{i-3}, \xi_{i-3}\right)\right],
$$

em que $i=3, \ldots, N-1$. Note que $\xi_{1}, \xi_{2}, \xi_{3}$ não estão definidas na equação (7), fazendo com que tais valores devam ser obtidos por algum outro método. Neste estudo utilizou-se o Runge-Kutta 
clássico definido na equação (5). O intervalo de estabilidade absoluta é dado por $J=(-0,3,0)$ [6].

Durante as pesquisas apresentadas neste trabalho, escolheu-se também trabalhar com os seguintes métodos implícitos.

\subsection{Método de Adams-Moulton 2}

No esquema de Adams-Moulton implícito, de dois pontos e de segunda ordem, a solução aproximada $\xi_{i+1}$ é definida por

$$
\xi_{i+1}=\xi_{i}+\frac{h}{2}\left[f\left(t_{i+1}, \xi_{i+1}\right)+f\left(t_{i}, \xi_{i}\right)\right], \quad i=0, \ldots, N-1 .
$$

Este procedimento também é conhecido como método Trapezoidal implícito. Note que neste método não é possível deixar $\xi_{i+1}$ de maneira que se possa obtê-la recursivamente (iterativo). Isso faz com que um sistema linear associado às equações (8) deva ser resolvido, para se encontrar os valores $\xi_{i}, i=1, \ldots, N-1$. O intervalo de estabilidade absoluta é dado por $J=(-\infty, 0)$ [6].

Uma alternativa para não precisar resolver o sistema linear é utilizar a técnica de predição e correção. Esta técnica consiste em estimar $\xi_{i+1}$, que está do lado direito de (8), por meio de um esquema explícito e substituí-lo na expressão que define o método implícito. Dessa maneira, o método de Adams-Moulton 2 preditor-corretor, cuja predição é realizada pelo método de AdamsBashforth 2, torna-se:

$$
\left\{\begin{array}{l}
\xi_{\text {pred }}=\xi_{i}+\frac{h}{2}\left[3 f\left(t_{i}, \xi_{i}\right)-f\left(t_{i-1}, \xi_{i-1}\right)\right] \\
\xi_{i+1}=\xi_{i}+\frac{h}{2}\left[f\left(t_{i+1}, \xi_{\text {pred }}\right)+f\left(t_{i}, \xi_{i}\right)\right]
\end{array}, i=1, \ldots, N-1\right.
$$

Ao executar tal procedimento, o intervalo de estabilidade absoluta se altera para $J=(-2,0)[6]$.

\subsection{Método de Adams-Moulton 4}

O esquema de Adams-Moulton implícito, de quatro pontos e de quarta ordem, fornece a solução aproximada $\xi_{i+1}$ definida por

$$
\xi_{i+1}=\xi_{i}+\frac{h}{24}\left[9 f\left(t_{i+1}, \xi_{i+1}\right)+19 f\left(t_{i}, \xi_{i}\right)-5 f\left(t_{i-1}, \xi_{i-1}\right)+f\left(t_{i-2}, \xi_{i-2}\right)\right],
$$

em que $i=2, \ldots, N-1$ e o intervalo de estabilidade absoluta é dado por $J=(-3,0)$ [6].

Para se evitar a resolução do sistema linear definido pelas equações (10), considerou-se a técnica de predição-correção, em conjunto com o método de Adams-Bashforth 4 explícito, cuja expressão é dada por

$$
\left\{\begin{array}{l}
\xi_{\text {pred }}=\xi_{i}+\frac{h}{24}\left[55 f\left(t_{i}, \xi_{i}\right)-59 f\left(t_{i-1}, \xi_{i-1}\right)+37 f\left(t_{i-2}, \xi_{i-2}\right)-9 f\left(t_{i-3}, \xi_{i-3}\right)\right] \\
\xi_{i+1}=\xi_{i}+\frac{h}{24}\left[9 f\left(t_{i+1}, \xi_{\text {pred }}\right)+19 f\left(t_{i}, \xi_{i}\right)-5 f\left(t_{i-1}, \xi_{i-1}\right)+f\left(t_{i-2}, \xi_{i-2}\right)\right]
\end{array}\right.
$$

em que $i=3, \ldots, N-1$. Essa estratégia faz com que o intervalo de estabilidade absoluta se altere para $J=(-1,3,0)[6]$. 


\subsection{Ordem de convergência}

Uma maneira de se avaliar assintoticamente a ordem de convergência de uma solução aproximada, obtida de um esquema, é realizar estudos numéricos através de simulações que comparam a solução aproximada, com a analítica para distintos espaçamentos $h$.

Denotando a solução analítica do PVI no ponto $t=t_{i}$ por $x_{i}=x\left(t_{i}\right)$ e a solução aproximada, obtida em uma partição regular de espaçamento $h$, por $\xi_{i}^{(h)}$, o erro absoluto e o erro relativo cometidos por uma solução numérica são, respectivamente [7]:

$$
E_{A}=\max \left\{\left|x\left(t_{i}\right)-\xi_{i}^{(h)}\right|, i=1, \ldots, N-1\right\}
$$

$\mathrm{e}$

$$
E_{R}=\max \left\{\frac{\left|x\left(t_{i}\right)-\xi_{i}^{(h)}\right|}{\left|x\left(t_{i}\right)\right|}, i=1, \ldots, N-1\right\} .
$$

Supondo que o método em questão seja convergente, espera-se que o erro absoluto (12) ou relativo (13) tenha um comportamento assintótico da forma

$$
E=C . h^{p} \Leftrightarrow \log (E)=\log (C)+p \log (h),
$$

onde $C>0$ e $p$ é a ordem de convergência.

Note que a equação (14) independe dos valores escolhidos para o espaçamento, quando este pertence à região de estabilidade do método em estudo. Dessa forma, simulações para diferentes valores de $h$ resultam nos respectivos erros e portanto, formam-se os pontos $\left(h_{i}, E_{i}\right)$. A regressão linear ajustada a estes pontos, pelo método dos Quadrados Mínimos, fornece condições para se obter os parâmetros $p$ e $C$, representados na equação (14). Neste trabalho foram realizados estudos numéricos da ordem de convergência dos métodos descritos anteriormente e os resultados estão expostos na Seção 3.

\section{Simulações numéricas}

Os métodos numéricos descritos na Seção 2 foram aplicados em três problemas de dinâmica populacional, com o intuito de analisar o comportamento das soluções aproximadas, à medida que diminui-se o espaçamento $h$. Além disso, as soluções foram comparadas entre si e, quando possível, foram também comparadas com a solução analítica. No caso do primeiro exemplo, cuja solução exata pode ser obtida explicitamente, realizou-se o estudo numérico da ordem de convergência dos métodos descritos e citados anteriormente.

\subsection{Equação logística}

Um modelo matemático importante na dinâmica populacional é o modelo de crescimento de Verhulst, cuja taxa de variação da população é proporcional à própria população, com a adição de um termo inibidor que faz com que a longo prazo a população tenda a um valor constante. Uma 
maneira de representá-lo é por meio do seguinte Problema de Valor Inicial (PVI):

$$
\left\{\begin{array}{l}
\frac{d x}{d t}=a x-b x^{2} \\
x(0)=x_{0}
\end{array}\right.
$$

em que $a>0$ é a taxa de crescimento da população $x=x(t), b>0$ é um fator que atua na inibição do crescimento de $x$, através do encontro entre os indivíduos da mesma população e $x_{0}$ é a quantidade inicial de indivíduos.

A solução analítica do PVI (15) é dada por

$$
x(t)=\frac{a x_{0} e^{a t}}{a+b x_{0}\left(e^{a t}-1\right)} .
$$

A discretização do PVI (15) foi feita utilizando os métodos numéricos descritos na Seção 2, cuja soluções numéricas foram obtidas para diferentes espaçamentos $h$, comparadas entre si, e também com a solução exata (16), ver Figuras 1, 2 e 3. Além disso, conforme será detalhado mais adiante, efetuou-se um estudo numérico da ordem de convergência considerando os erros absolutos e relativos de cada esquema (ver Tabelas 1 e 2), em que as regressões lineares estão expostas nos gráficos da Figura 4. Para este exemplo tem-se $t \in[0,10], a=1, b=0,01$ e $x_{0}=2$, valores estes presentes na literatura [6].

Os gráficos da Figura 1 apresentam as soluções aproximadas obtidas para $N=8$ e $N=16$. Na primera linha, gráfico à esquerda, percebe-se visualmente que o método de Runge-Kutta Clássico (RK4) possui uma solução numérica mais próxima da solução analítica, do que as demais soluções, utilizando-se apenas 9 pontos. Por outro lado, no gráfico à direita, os métodos AdamsBashforth de dois pontos (Ad-Bash2) e Adams-Bashforth de quatro pontos (Ad-Bash4) possuem oscilações causadas pelo fato de o espaçamento $h=1,25$ não satisfazer suas respectivas condições de estabilidade, isto é, $\lambda h \notin J=(-1,0)$ e $\lambda h \notin J=(-0,3,0)$, respectivamente. Note que $\lambda$ é tal como foi definido na Seção 2.1 (neste exemplo, obteve-se $\lambda=-1$ ). Já no caso do método Adams-Moulton de quatro pontos preditor-corretor (Ad-Moulp4), $\lambda h \in J=(-1,3,0)$, entretanto há pequenas oscilações em razão de $h=1,25$ estar próximo da fronteira do seu intervalo de estabilidade. Além disso, os métodos de Euler, Euler Modificado (Euler Mod) e Adams-Moulton de dois pontos preditor-corretor (Ad-Moulp2) possuem uma solução aproximada estável para $N=8$.

A partir de $N=16$, Figuras 2 e 3, todos os métodos possuem soluções que visualmente assemelham-se a solução exata e as soluções aproximadas ficam cada vez mais próximas da solução analítica à medida que o espaçamento $h$ tende para zero. Os gráficos da segunda linha da Figura 1 mostram que, visualmente, os métodos do tipo Adams possuem soluções próximas da solução analítica.

Na Figura 2, na segunda linha quando $N=64$, com exceção do método de Euler, todos os esquemas possuem solução numérica visualmente próxima da solução analítica, a ponto de não ser possível observar diferença entre tais soluções. Observando as soluções determinadas pelo método de Euler para $N=8, N=16, N=32$ e $N=64$ (Figuras 1 e 2) nota-se uma convergência mais lenta para a solução exata, em comparação com os demais métodos.

A medida que se diminui o espaçamento, as diferenças entre as soluções numéricas e exata ficam visualmente imperceptíveis. Com $N=128$, Figura 3 primeira linha, tem-se o método de 

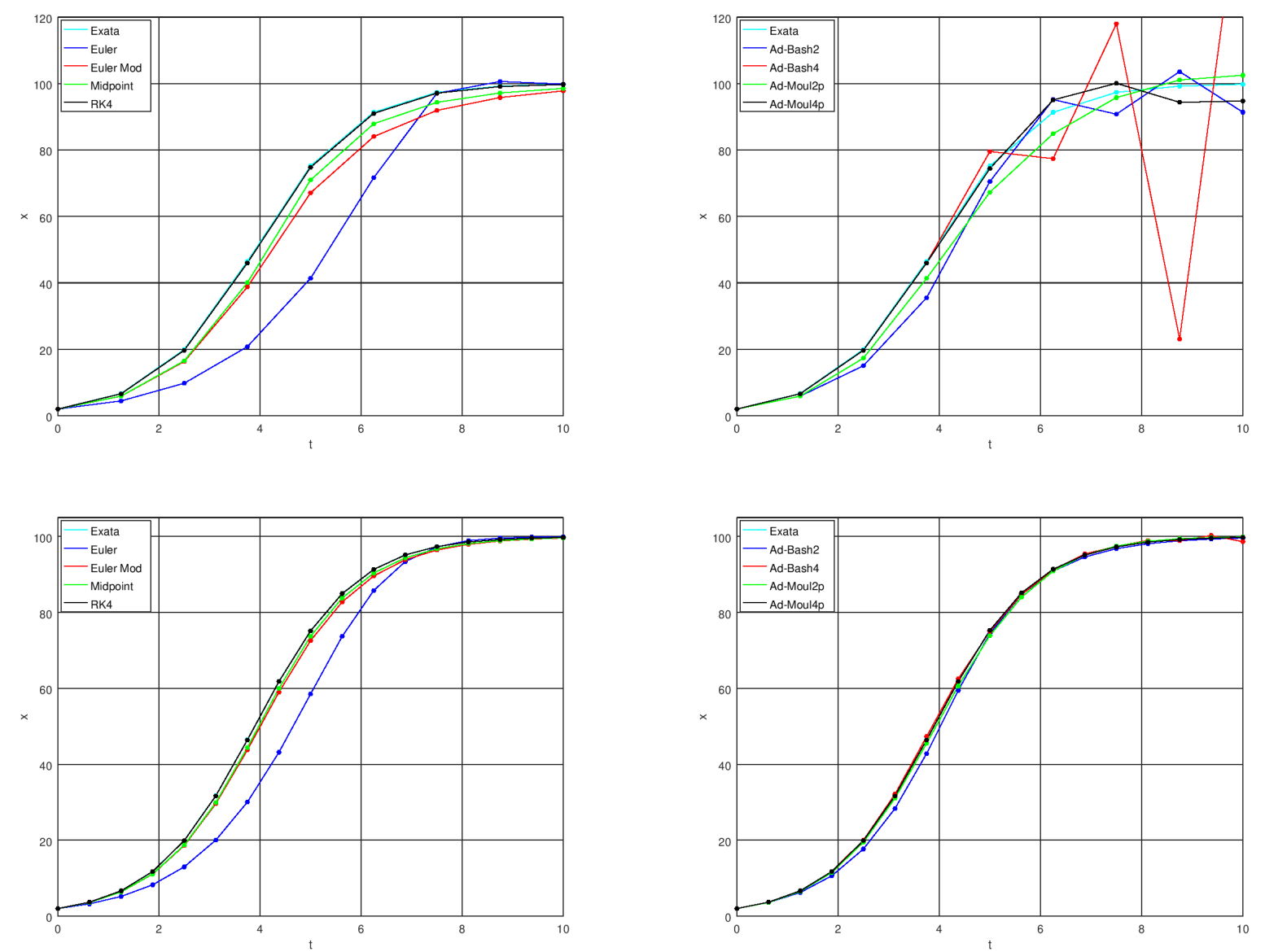

Figura 1: Comparação entre as soluções aproximadas e a solução exata. Na primeira linha tem-se $h=1,25(\mathrm{~N}=8)$ e na segunda linha $h=0,625(\mathrm{~N}=16)$. 

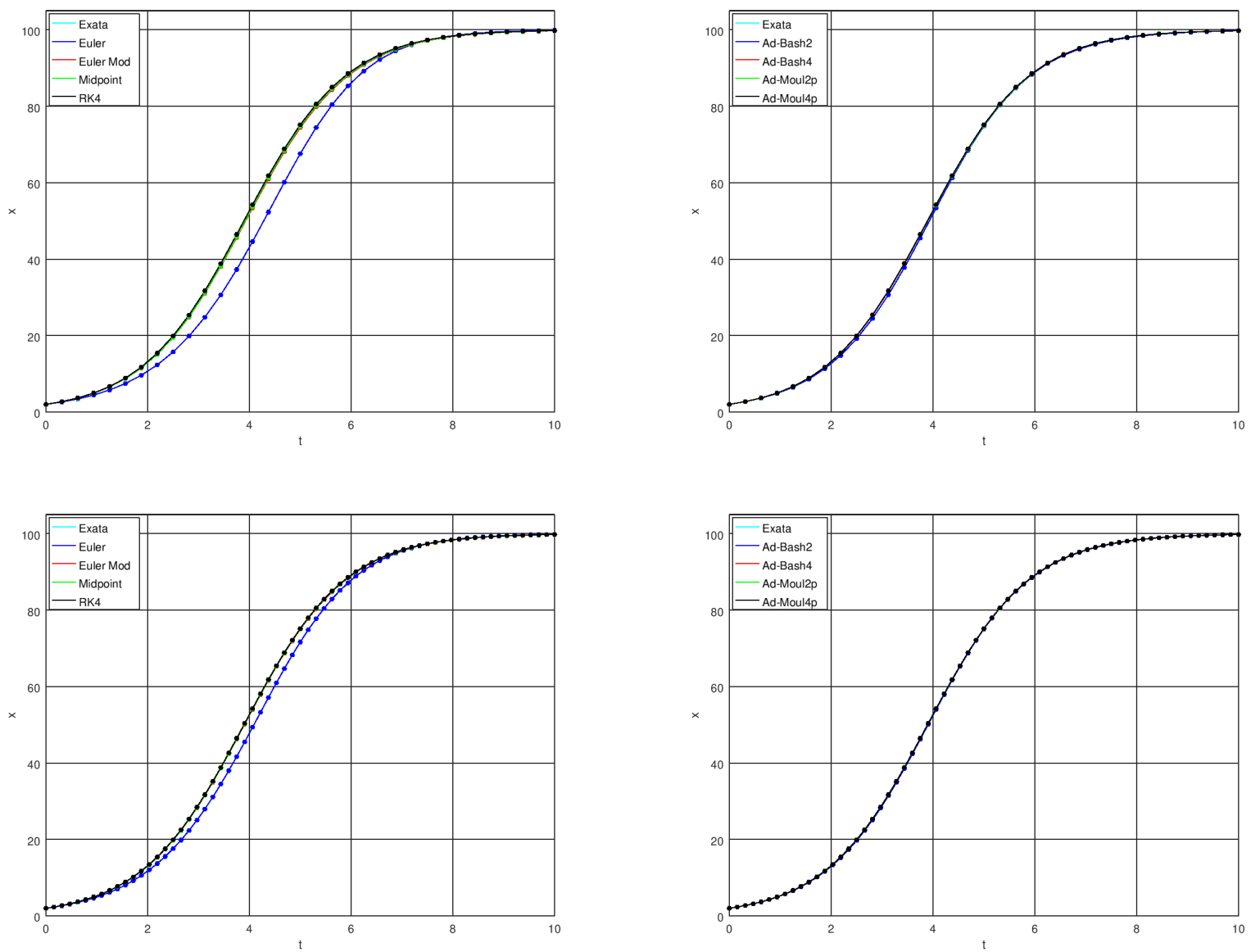

Figura 2: Comparação entre as soluções aproximadas e a solução exata. Na primeira linha tem-se $h=0,3125(\mathrm{~N}=32)$ e na segunda linha $h=0,15625(\mathrm{~N}=64)$. 
Euler bem próximo da solução analítica e $\operatorname{com} N=1024$ praticamente não se vê mais diferenças entre as soluções.
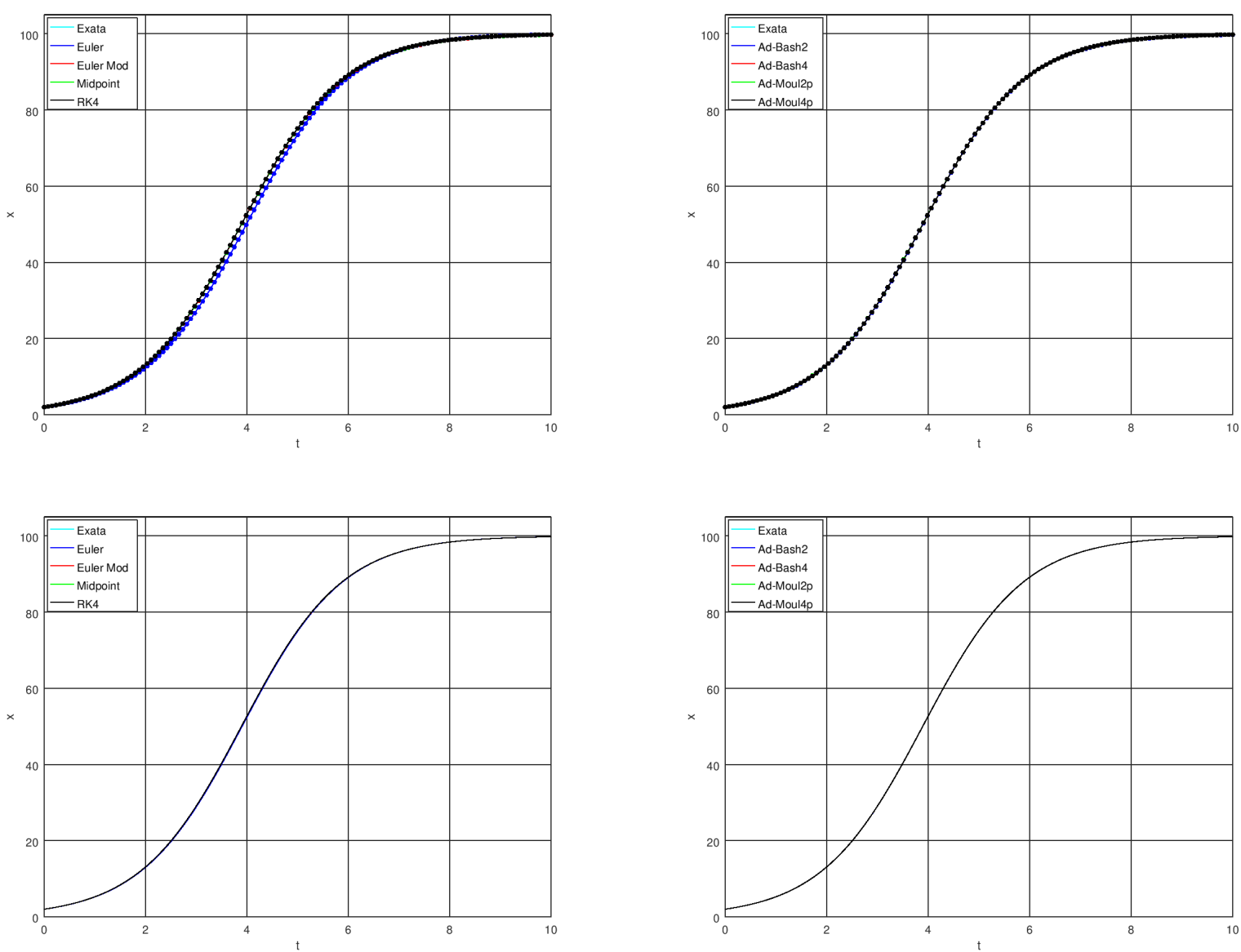

Figura 3: Comparação entre as soluções aproximadas e a solução exata. Na primeira linha tem-se $h=0,078125(\mathrm{~N}=128)$ e na segunda linha $h=0,009765625(\mathrm{~N}=1028)$.

Embora as Figuras 1, 2 e 3 mostrem visualmente que as soluções numéricas estão convergindo para a solução analítica e que há diferenças na maneira como cada método converge, uma forma mais eficaz de fazer tal análise é por meio do estudo da ordem de convergência, proposto na Seção 2, utilizando o erro absoluto $\left(E_{A}\right)$ e o erro relativo $\left(E_{R}\right)$.

Nas Tabelas 1 e 2 encontram-se os erros absolutos e relativos a medida que o espaçamento $h$ diminui. Efetuando a regressão linear dos valores que estão nas tabelas, a partir de $N=16$, na última linha encontram-se as ordens de convergências obtidas para cada método. Assim, concluiu-se que o método de Euler é de primeira ordem, os métodos Euler modificado, Midpoint, Adams-Bashforth 2 e Adams-Moulton 2 são de segunda ordem e os métodos Adams-Bashforth 4 e Adams-Moulton 4 são de quarta ordem.

Esses valores correspondem à inclinação $p$ das retas que estão nos gráficos da Figura 4. Dessa forma, nota-se as diferenças com que as soluções aproximadas de cada método convergem para 
a solução analítica, tanto pelos valores das Tabelas 1 e 2 quanto pelas representações gráficas da ordem de convergência, através das retas ajustadas, Figura 4.

Tabela 1: Erro Absoluto, Erro Relativo e Ordem de convergência.

\begin{tabular}{|c|c|c|c|c|c|c|c|c|}
\hline \multirow[t]{2}{*}{$\mathrm{N}$} & \multicolumn{2}{|c|}{ Euler } & \multicolumn{2}{|c|}{ Euler Mod } & \multicolumn{2}{|c|}{ Midpoint } & \multicolumn{2}{|c|}{ RK4 } \\
\hline & $\overline{E_{a}}$ & $E_{r}$ & $E_{a}$ & $\overline{E_{r}}$ & $E_{a}$ & $E_{r}$ & $\overline{E_{a}}$ & $E_{r}$ \\
\hline 8 & 33.823 & 0.1291 & 8.0846 & 0.0606 & 6.4195 & 0.0354 & 0.4398 & 0.0027 \\
\hline 16 & 18.682 & 0.0633 & 2.8343 & 0.0150 & 2.0511 & 0.0096 & 0.0400 & $2.02 \mathrm{e}-04$ \\
\hline 32 & 9.6560 & 0.0311 & 0.8370 & 0.0041 & 0.5955 & 0.0027 & 0.0031 & $1.45 e-05$ \\
\hline 64 & 4.9123 & 0.0154 & 0.2291 & 0.0011 & 0.1618 & $7.17 \mathrm{e}-04$ & $2.14 \mathrm{e}-04$ & $9.84 \mathrm{e}-07$ \\
\hline 128 & 2.4720 & 0.0077 & 0.0600 & $2.79 \mathrm{e}-04$ & 0.0423 & $1.86 \mathrm{e}-04$ & $1.41 \mathrm{e}-05$ & $6.42 \mathrm{e}-08$ \\
\hline 256 & 1.2396 & 0.0038 & 0.0154 & $7.11 \mathrm{e}-05$ & 0.0108 & $4.75 e-05$ & $9.02 \mathrm{e}-07$ & $4.10 \mathrm{e}-09$ \\
\hline 512 & 0.6207 & 0.0019 & 0.0039 & $1.79 \mathrm{e}-05$ & 0.0027 & $1.20 \mathrm{e}-05$ & $5.71 \mathrm{e}-08$ & $2.59 \mathrm{e}-10$ \\
\hline 1024 & 0.3106 & $9.54 \mathrm{e}-04$ & $9.78 \mathrm{e}-04$ & $4.51 \mathrm{e}-06$ & $6.87 \mathrm{e}-04$ & $3.02 \mathrm{e}-06$ & $3.59 \mathrm{e}-09$ & $1.63 \mathrm{e}-11$ \\
\hline 2048 & 0.1553 & $4.77 \mathrm{e}-04$ & $2.45 \mathrm{e}-04$ & $1.13 \mathrm{e}-06$ & $4.51 \mathrm{e}-04$ & $7.57 \mathrm{e}-07$ & $2.25 \mathrm{e}-10$ & $1.02 \mathrm{e}-12$ \\
\hline Ordem & \multicolumn{2}{|c|}{1.00} & \multicolumn{2}{|c|}{1.96} & \multicolumn{2}{|c|}{1.95} & \multicolumn{2}{|c|}{3.94} \\
\hline
\end{tabular}

Tabela 2: Erro Absoluto, Erro Relativo e Ordem de convergência

\begin{tabular}{|c|c|c|c|c|c|c|c|c|}
\hline \multirow[t]{2}{*}{$\mathrm{N}$} & \multicolumn{2}{|c|}{ Ad-Bash2 } & \multicolumn{2}{|c|}{ Ad-Moulp2 } & \multicolumn{2}{|c|}{ Ad-Moulp4 } & \multicolumn{2}{|c|}{ Ad-Bash4 } \\
\hline & $E_{a}$ & $E_{r}$ & $E_{a}$ & $E_{r}$ & $E_{a}$ & $E_{r}$ & $E_{a}$ & $E_{r}$ \\
\hline$\overline{88}$ & 10.926 & 0.0702 & $\overline{7.8881}$ & 0.0454 & $\overline{5.0348}$ & 0.0364 & 76.122 & 0.3865 \\
\hline 16 & 3.6204 & 0.0096 & 1.2754 & 0.0059 & 0.1572 & $5.97 \mathrm{e}-04$ & 1.1281 & 0.0048 \\
\hline 32 & 1.0535 & 0.0031 & 0.1565 & $5.80 \mathrm{e}-04$ & 0.0051 & $1.96 \mathrm{e}-05$ & 0.0679 & $1.60 \mathrm{e}-04$ \\
\hline 64 & 0.2812 & $8.75 e-04$ & 0.0212 & $7.65 e-05$ & $2.55 \mathrm{e}-04$ & $8.83 e-07$ & 0.0047 & $1.04 \mathrm{e}-05$ \\
\hline 128 & 0.0726 & $2.32 \mathrm{e}-04$ & 0.0096 & $2.76 e-05$ & $1.91 \mathrm{e}-05$ & $5.03 e-08$ & $2.98 \mathrm{e}-04$ & $6.70 \mathrm{e}-07$ \\
\hline 256 & 0.0184 & $5.98 \mathrm{e}-05$ & 0.0031 & $9.49 \mathrm{e}-06$ & $1.30 \mathrm{e}-06$ & $3.13 e-09$ & $1.87 \mathrm{e}-05$ & $4.24 \mathrm{e}-08$ \\
\hline 512 & 0.0046 & $1.52 \mathrm{e}-05$ & $8.46 \mathrm{e}-04$ & $2.72 \mathrm{e}-06$ & $8.52 \mathrm{e}-08$ & $1.98 \mathrm{e}-10$ & $1.17 \mathrm{e}-06$ & $2.67 \mathrm{e}-09$ \\
\hline 1024 & 0.0012 & $3.81 \mathrm{e}-06$ & $2.22 \mathrm{e}-04$ & $7.24 \mathrm{e}-07$ & $5.44 \mathrm{e}-09$ & $1.25 \mathrm{e}-11$ & $7.34 \mathrm{e}-08$ & $1.67 \mathrm{e}-10$ \\
\hline 2048 & $2.92 \mathrm{e}-04$ & $9.57 \mathrm{e}-07$ & $5.70 \mathrm{e}-05$ & $1.86 \mathrm{e}-07$ & $3.43 e-10$ & $7.88 \mathrm{e}-13$ & $4.59 \mathrm{e}-09$ & $1.05 \mathrm{e}-11$ \\
\hline Ordem & \multicolumn{2}{|c|}{1.91} & \multicolumn{2}{|c|}{1.99} & \multicolumn{2}{|c|}{4.16} & \multicolumn{2}{|c|}{4.05} \\
\hline
\end{tabular}

\subsection{Modelo de Lotka-Volterra}

O sistema de equações que descrevem o modelo de Lotka-Volterra é um problema clássico de presa-predador, que pode ser aplicável em sistemas biológicos [10] para análise das populações envolvidas. Tal modelo é representado matematicamente pelo seguinte PVI:

$$
\left\{\begin{array}{l}
\frac{d P_{1}}{d t}=a P_{1}-b P_{1} P_{2} \\
\frac{d P_{2}}{d t}=-c P 2+d P_{1} P_{2} \\
P_{1}(0)=p_{1_{0}} \text { e } P_{2}(0)=p_{2_{0}}
\end{array}\right.
$$



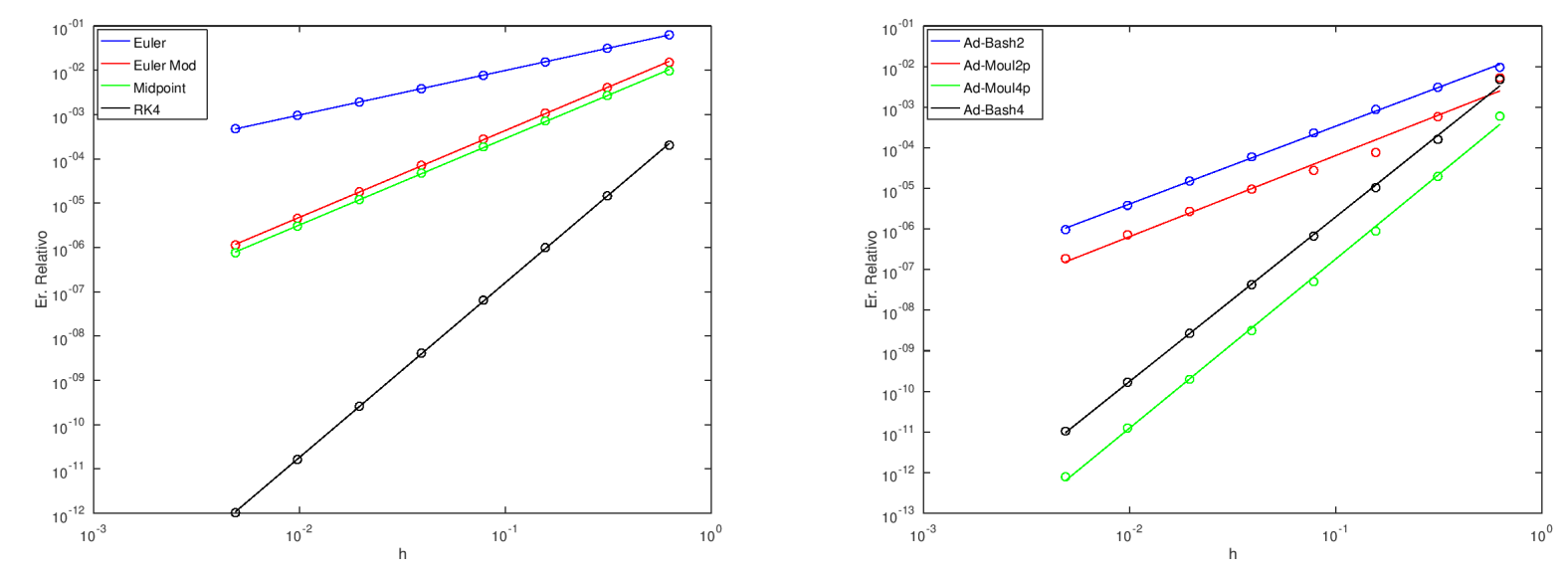

Figura 4: Estudo Numérico da ordem de convergência de cada método investigado.

donde $P_{1}$ é a população de presas em um ambiente em que há condições suficientes para o crescimento das presas, a uma taxa representada por $a, P_{2}$ é a população de predadores que se alimentam de $P_{1}$ e por esse motivo se beneficiam do encontro entre $P_{1}$ e $P_{2}$, representado pela multiplicação $P_{1} P_{2}$, e consequentemente, limitam o crescimento indefinido de $P_{1}$. O coeficiente $a$ é a taxa de crescimento das presas, $c$ a taxa de mortalidade dos predadores, $b$ a taxa de mortes no encontro entre $P_{1}$ e $P_{2}$ e $d$ a taxa com que o alimento influencia no crescimento de $P_{2}$. Os valores $p_{1_{0}}$ e $p_{2_{0}}$ são as quantidades iniciais de presa e predador.

O PVI (17) possui apenas uma solução geral analítica implícita da seguinte forma [11]

$$
a \ln (y)-b y+c \ln (x)-d x=C,
$$

em que $C$ é uma constante de integração, $x=P_{1}$ e $y=P_{2}$.

De maneira geral, pela análise dos pontos críticos associados ao PVI (17), as soluções são curvas fechadas em torno do ponto crítico central $\left(\frac{c}{d}, \frac{a}{b}\right)$, com exceção do ponto crítico $(0,0)$. Assim, no plano de fase, as populações têm uma variação cíclica para $a, b, c, d>0$ [11]. Essa conclusão também pode ser alcançada por meio de uma análise dos autovalores associados à formulação matricial do PVI (17), sem a necessidade de se resolver analiticamente o PVI [12].

Aplicando os métodos numéricos da Seção 2, foram determinadas as soluções aproximadas representadas nas Figuras 5, 7 e 9, além dos respectivos planos de fase, Figuras 6, 8 e 10, visando auxiliar na análise das soluções numéricas. Neste exemplo foram adotados os valores $a=2$, $b=1, c=1, d=1, p_{1_{0}}=1, p_{2_{0}}=0,1$ e $t_{f}=20$, que correspondem a aproximadamente três ciclos no plano de fase [6].

Na Figura 5 tem-se as soluções numéricas de $P_{1}$ e $P_{2}$ e na Figura 6 seus respectivos ciclos. Ao observar os métodos de passo simples, primeira coluna da Figura 5, verifica-se que a cada ciclo, o pico das populações aumenta de valor enquanto que tais picos permanecem constantes nas soluções dos métodos de múltiplos passos, segunda coluna da Figura 5. Uma consequência desse aumento é que, a cada ciclo, a curva no plano de fase se afasta do ponto central $(1,2)$, Figura 6, contrariando a propriedade do comportamento cíclico das trajetórias do plano de fase associado ao sistema presa-predador descrito pelo sistema (17). 

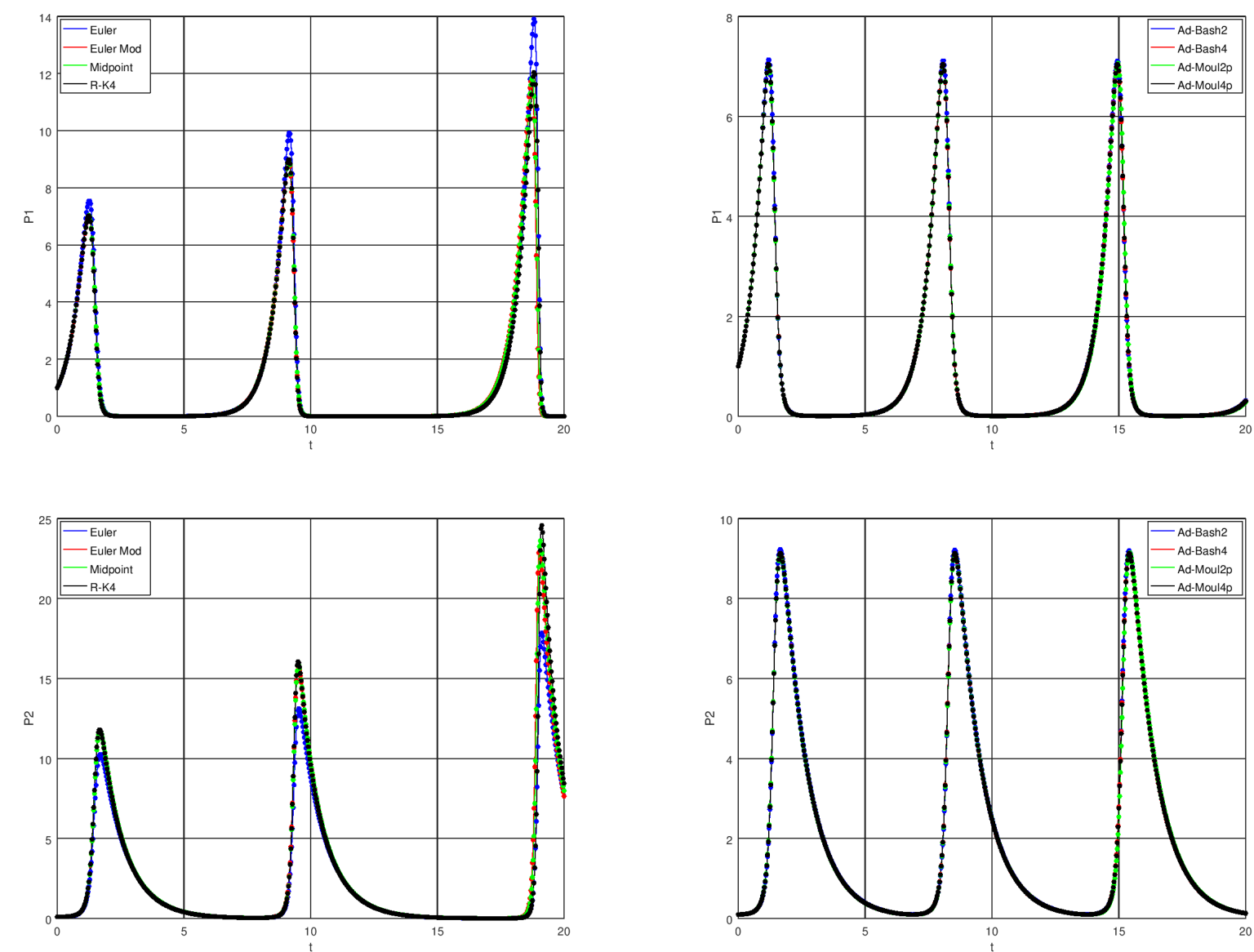

Figura 5: Comparação entre as soluções aproximadas para $h=0,03125$ (N=640) e tempo final $t_{f}=20$. Na primeira linha tem-se soluções para $P_{1}$ e na segunda linha para $P_{2}$. 
Ao diminuir o espaçamento $h$, Figuras 7 e 9, nota-se que os picos das populações ficam cada vez menores, à medida que a quantidade de pontos na partição regular é aumentada. Por outro lado, mesmo com $N=5120$ (Figura 9), ainda percebe-se um aumento no pico a medida que a evolução temporal muda de ciclo, e tal comportamento fica nítido na Figura 10.
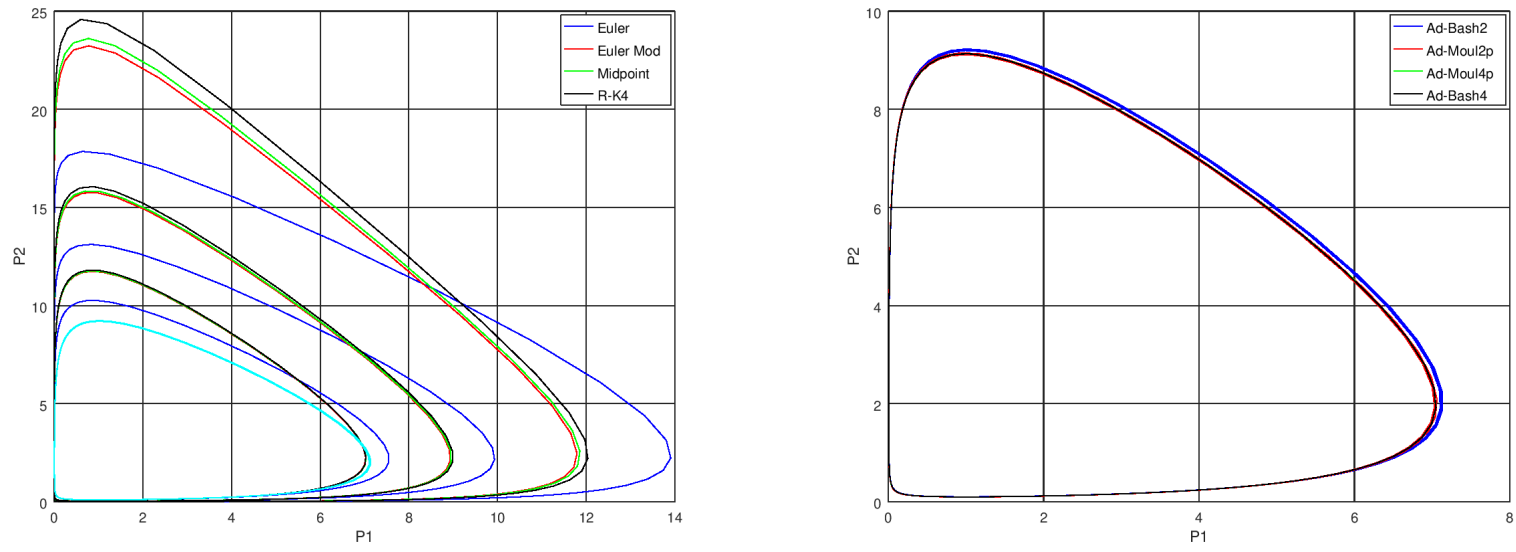

Figura 6: Plano de fase para $P_{1}$ e $P_{2} \operatorname{com} h=0,03125(\mathrm{~N}=640)$ e tempo final $t_{f}=20$. A curva de cor azul claro do gráfico à esquerda equivale a trajetória obtida pelo Método Ad-Moult4p que também está no gráfico à direita.

Considerando as figuras geradas neste exemplo, Figuras 5, 6, 7, 8, 9 e 10, constata-se a dificuldade que os métodos explíticos de passo simples de Euler, Euler Modificado, Midpoint e Runge-Kutta Clássico têm para obter soluções aproximadas em problemas do tipo Lotka-Volterra. Em contrapartida, os métodos de múltiplos passos lidam melhor com problemas deste tipo. Ao considerar todas as trajetórias dos métodos de passo simples, é importante destacar que o método de Euler foi o que apresentou maior proximidade visual com a trajetória do método de AdamsMoulton 4 (curva de cor azul claro no gráfico à esquerda das Figuras 6, 8 e 10), mesmo o método de Euler possuindo ordem de convergência menor.

Uma questão essencial é investigar a razão pela qual este exemplo difere do primeiro (visto na Seção 3.1), no sentido de que, geralmente, espera-se que métodos explícitos de maior ordem de precisão forneçam soluções mais próximas da solução analítica, para um mesmo $h$ e isto não aconteceu com os métodos explícitos aplicados ao modelo de Lotka-Volterra deste exemplo. Um caminho apontado na literatura leva ao conceito de problema rígido (stiff problem).

Embora o conceito de problema rígido seja impreciso e não tenha uma definição rigorosa [6], é comum considerar como stiff problems aqueles problemas nos quais os métodos explícitos não trabalham bem ou produzem resultados inesperados [13].

Neste exemplo envolvendo o modelo de Lotka-Volterra e os parâmetros escolhidos, ao se utilizar métodos explícitos de passo simples obteve-se dois resultados inesperados: i) o método explícito de passo simples, de menor ordem de convergência (método de Euler) mostrando soluções mais próximas da solução de referência e ii) aumento no pico das populações a cada ciclo, contrariando o comportamento cíclico das trajetórias do plano de fase associado ao sistema 

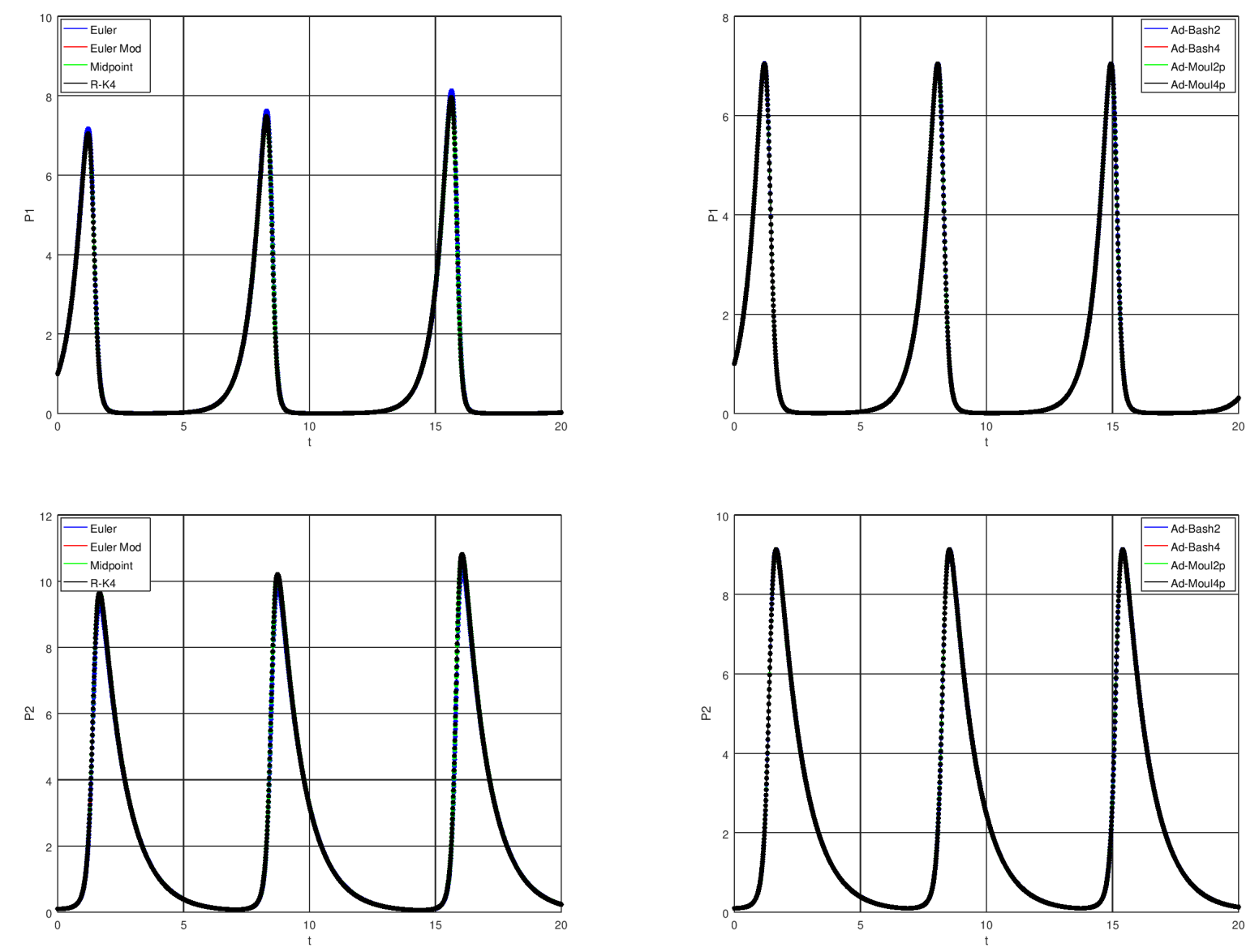

Figura 7: Comparação entre as soluções aproximadas para $h=0,0078125(\mathrm{~N}=2560)$ e tempo final $t_{f}=20$. Na primeira linha tem-se soluções para $P_{1}$ e na segunda linha para $P_{2}$. 

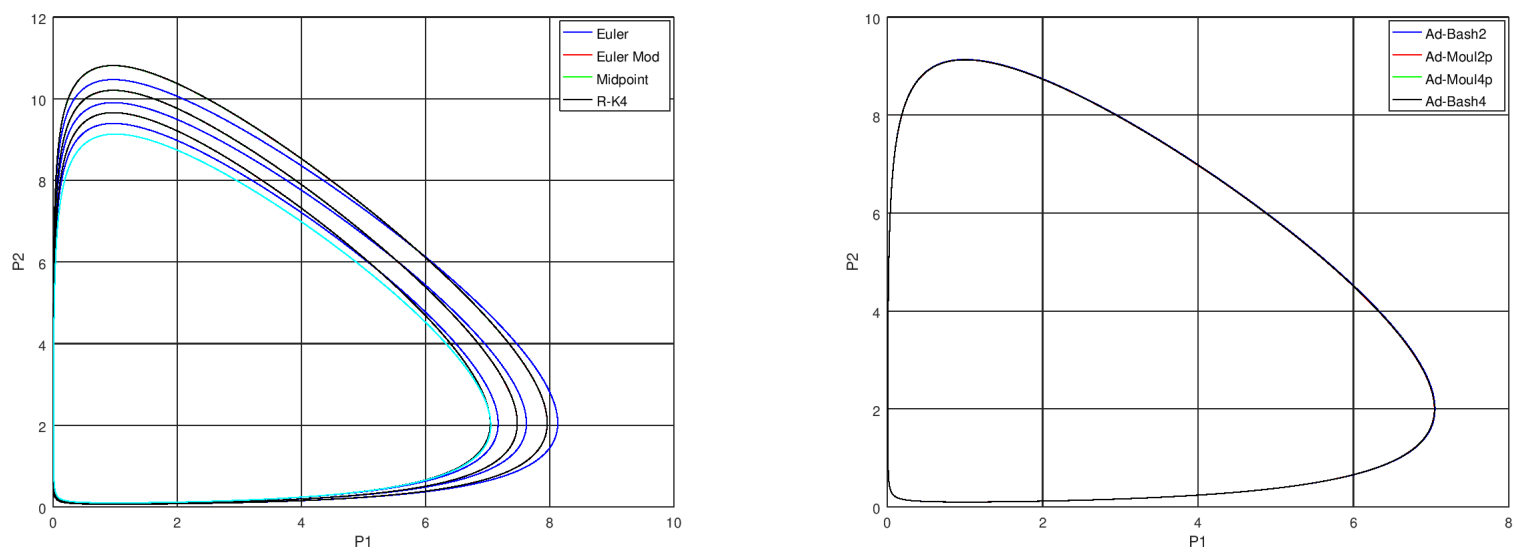

Figura 8: Plano de fase para $P_{1}$ e $P_{2} \operatorname{com} h=0,0078125(\mathrm{~N}=2560)$ e tempo final $t_{f}=20$.

presa-predador, conforme já comentado anteriormente. Portanto, este exemplo é um problema rígido.

A análise de desempenho de métodos numéricos aplicados em problemas rígidos vem sendo pesquisada e ainda não há um procedimento fechado. Contudo, sabe-se que além da ordem de convergência, outros aspectos devem ser levados em conta como região de estabilidade absoluta, se o sistema é periódico e a diferença entre as magnitudes dos autovalores associados ao sistema de EDO, entre outros $[13,6]$. Logo, não é garantido que uma maior ordem de convergência em métodos explícitos irá implicar em soluções numéricas mais próximas da solução analítica. No geral, recomenda-se o uso de métodos implícitos.

De fato, nas simulações realizadas (Figuras 5, 6, 7, 8, 9 e 10, gráficos à direita das figuras) os métodos implícitos Adams-Moulton 2 e 4 apresentaram os resultados mais satisfatórios, quando comparados aos demais. Isto é, a periodicidade dos ciclos foi mantida.

\subsection{Interação entre três espécies}

Este problema representa a dinâmica populacional de três espécies [14, 15], baseado no sistema presa-predador, cujo PVI é dado por

$$
\left\{\begin{array}{l}
\frac{d P_{1}}{d t}=P_{1}\left(1-0,001 P_{1}-0,001 P_{2}-0,015 P_{3}\right) \\
\frac{d P_{2}}{d t}=P_{2}\left(1-0,0015 P_{1}-0,001 P_{2}-0,001 P_{3}\right) \\
\frac{d P_{3}}{d t}=P_{3}\left(-1+\alpha P_{1}+0,0005 P_{2}\right) \\
P_{1}(0)=p_{1_{0}}, P_{2}(0)=p_{2_{0}} \text { e } P_{3}(0)=p_{3_{0}}
\end{array},\right.
$$

em que $P_{1}$ e $P_{2}$ são presas de $P_{3}$ o predador. 

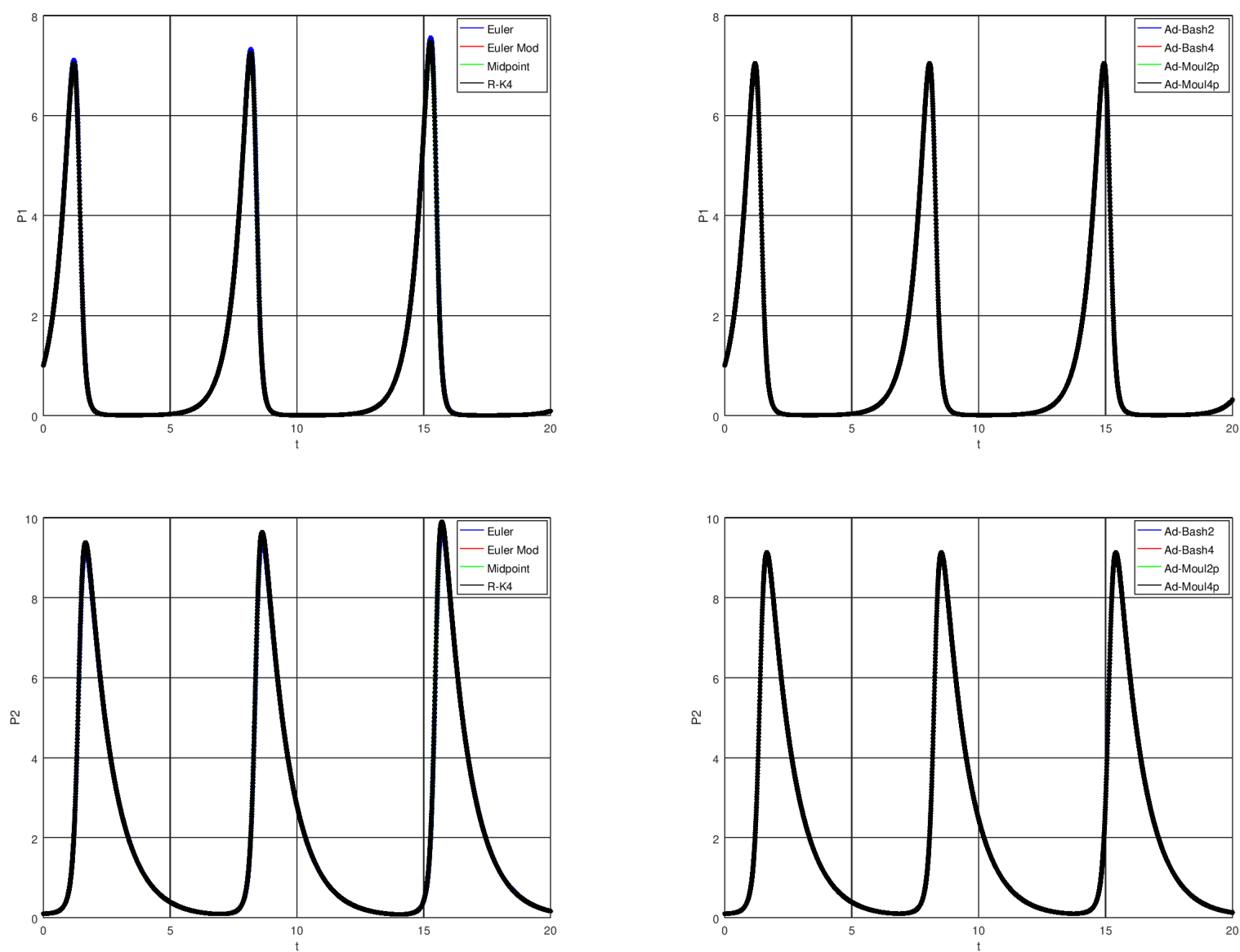

Figura 9: Comparação entre as soluções aproximadas para $h=0,00390625(\mathrm{~N}=5120)$ e tempo final $t_{f}=20$. Na primeira linha tem-se soluções para $P_{1}$ e na segunda linha para $P_{2}$. 

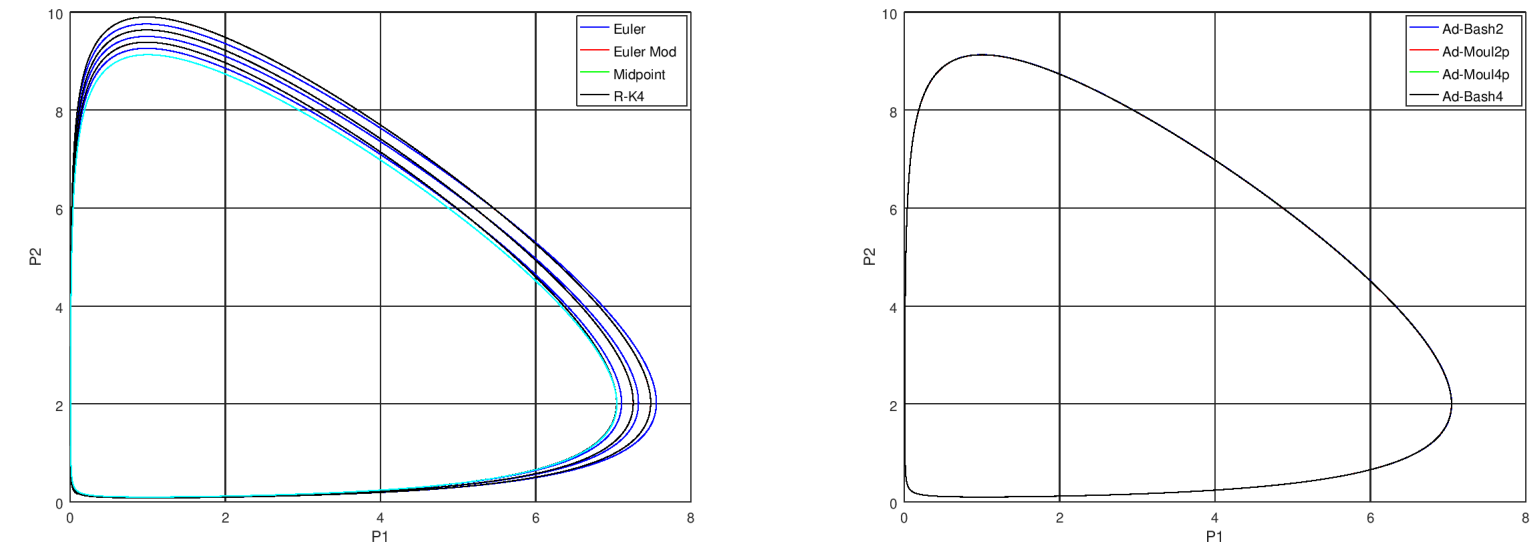

Figura 10: Plano de fase para $P_{1}$ e $P_{2} \operatorname{com} h=0,00390625(\mathrm{~N}=5120)$ e tempo final $t_{f}=20$. A curva de cor azul claro do gráfico à esquerda equivale a trajetória obtida pelo método Ad-Moult4p que também está no gráfico à direita.

Quando $\alpha=0,002$ as espécies evoluem para um ponto de equilíbrio estável, isto é, as populações tendem para um valor constante quando $t$ tende para infinito $[12,14,15]$. Considerando os valores iniciais $p_{1_{0}}=300, p_{2_{0}}=300$ e $p_{3_{0}}=50$, simulações foram executadas para $t f=200$ com $N=800$, isto é, $h=0,25$, e obteve-se as soluções aproximadas para $P_{1}, P_{2}$ e $P_{3}$, conferir Figuras 11, 12 e 13, respectivamente.
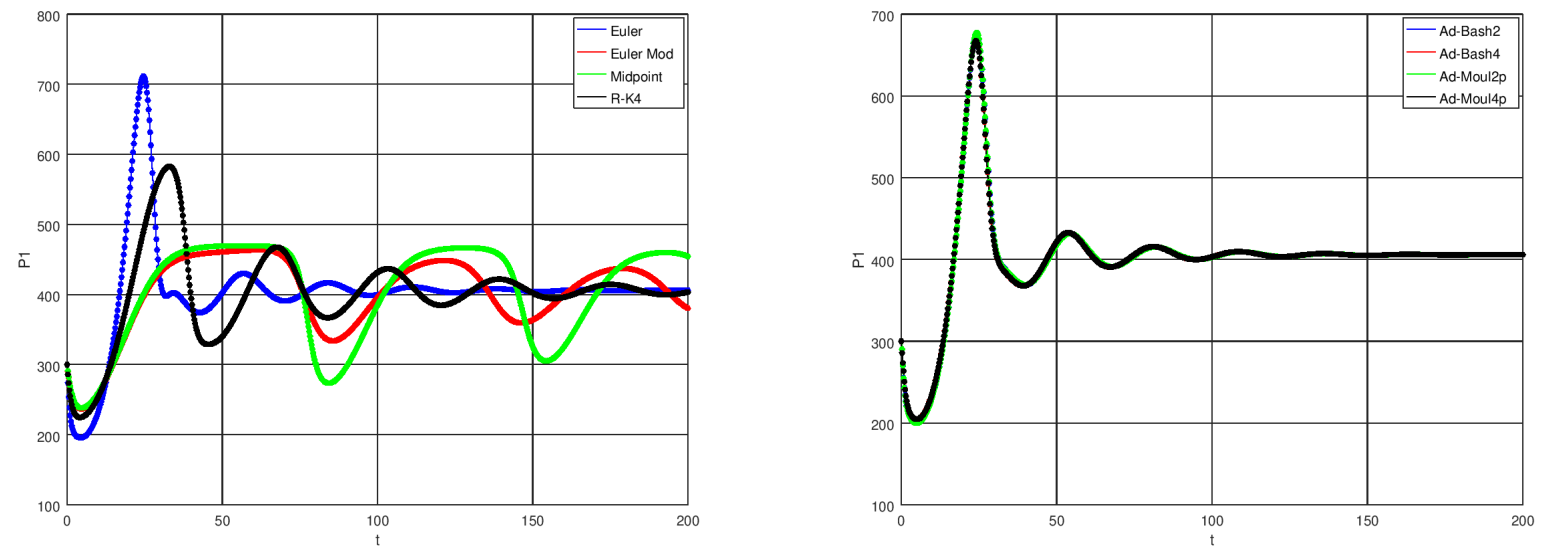

Figura 11: Comparação entre as soluções aproximadas para $h=0,25(\mathrm{~N}=800)$ e tempo final $t_{f}=20$ para a população $P_{1}$.

Conclui-se que os métodos de passo simples apontam para diferentes soluções aproximadas, ver gráficos à esquerda das Figuras 11, 12 e 13, já os métodos de passos múltiplos concordam visualmente com o formato das soluções para $P_{1}, P_{2}$ e $P_{3}$, ver gráficos à direita. Consequentemente, 

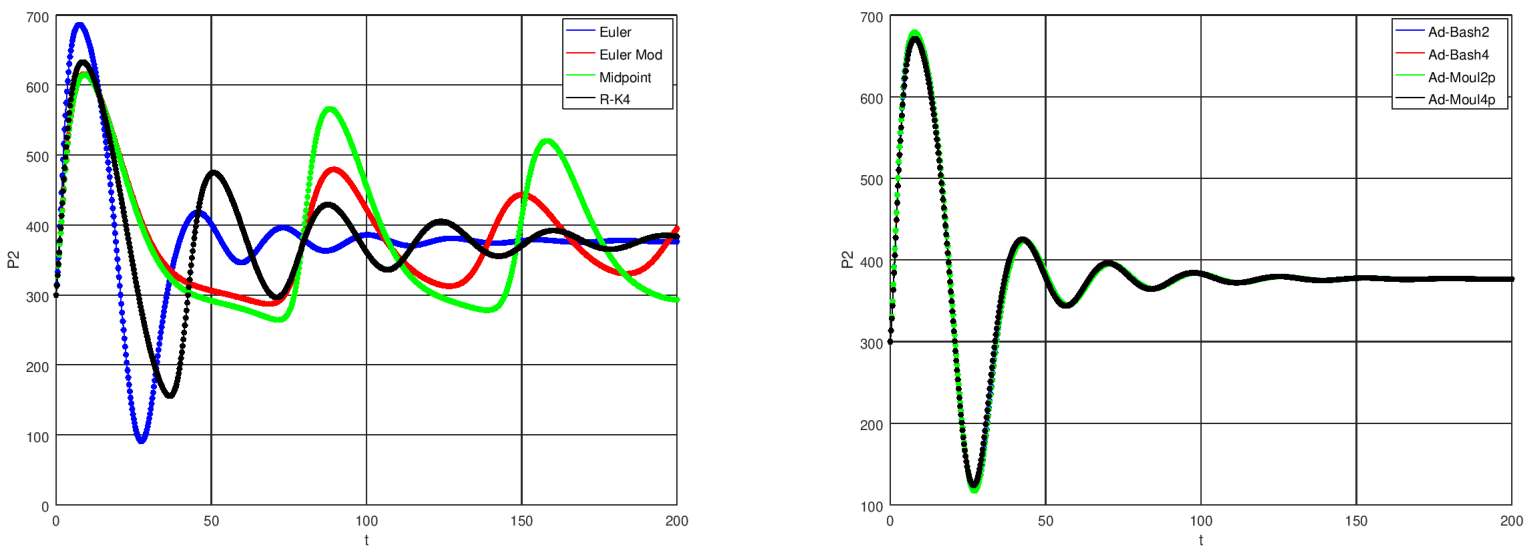

Figura 12: Comparação entre as soluções aproximadas para $h=0,25(\mathrm{~N}=800)$ e tempo final $t_{f}=20$ para a população $P_{2}$.
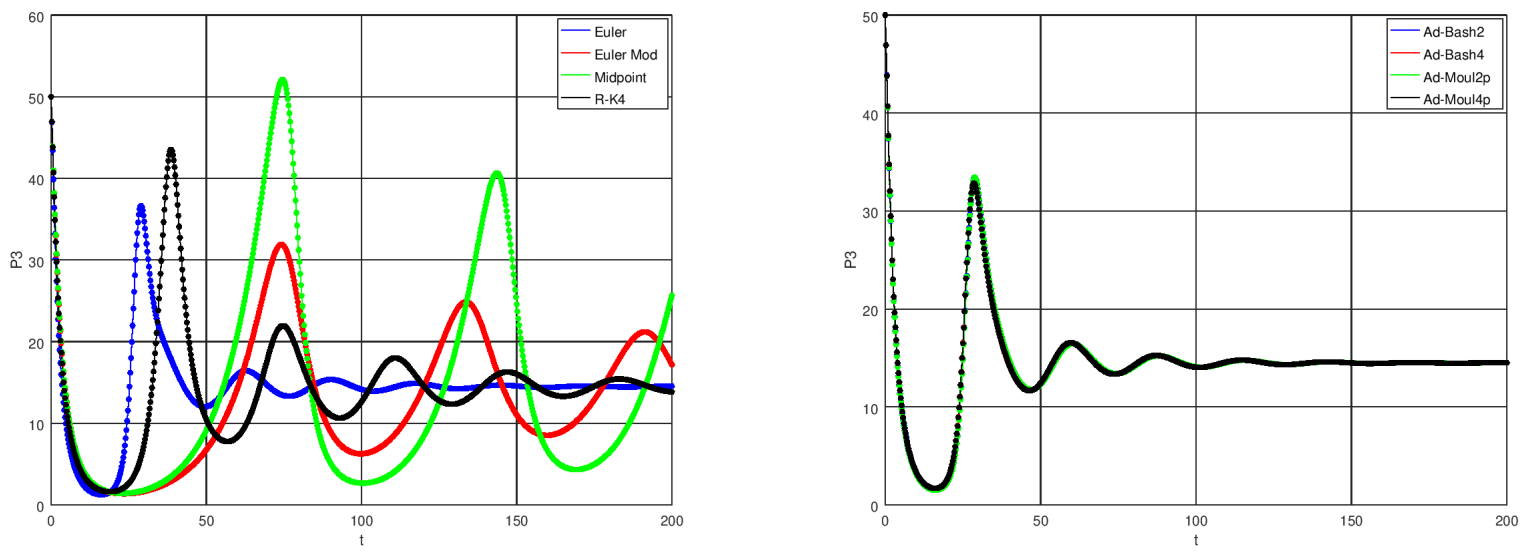

Figura 13: Comparação entre as soluções aproximadas para $h=0,25(\mathrm{~N}=800)$ e tempo final $t_{f}=20$ para a população $P_{3}$. 
o espaço de fase dos métodos de passo simples discordam com a forma da espiral, entretanto, todas as soluções aproximadas evoluem para o mesmo ponto de equilíbrio estável, enquanto que as espirais dos métodos de múltiplos passos possuem o mesmo formato, conferir Figura 14. Contudo, um fato significativo à se destacar é que, ao comparar a trajetória obtida pelo método de Euler, com a trajetória do método de Adams-Moulton 4 (curva de cor azul claro no gráfico à direita da Figura 14), as duas estão mais próximas visualmente. Quando comparadas as demais trajetórias dos métodos de passo simples, com o mesmo Adams-Moulton 4, estas ficam mais distantes, mesmo com ordens de convergência maiores do que o método de Euler. Conforme detalhado no exemplo anterior, este caso também pode ser classificado como problema rígido (ou stiff problem).
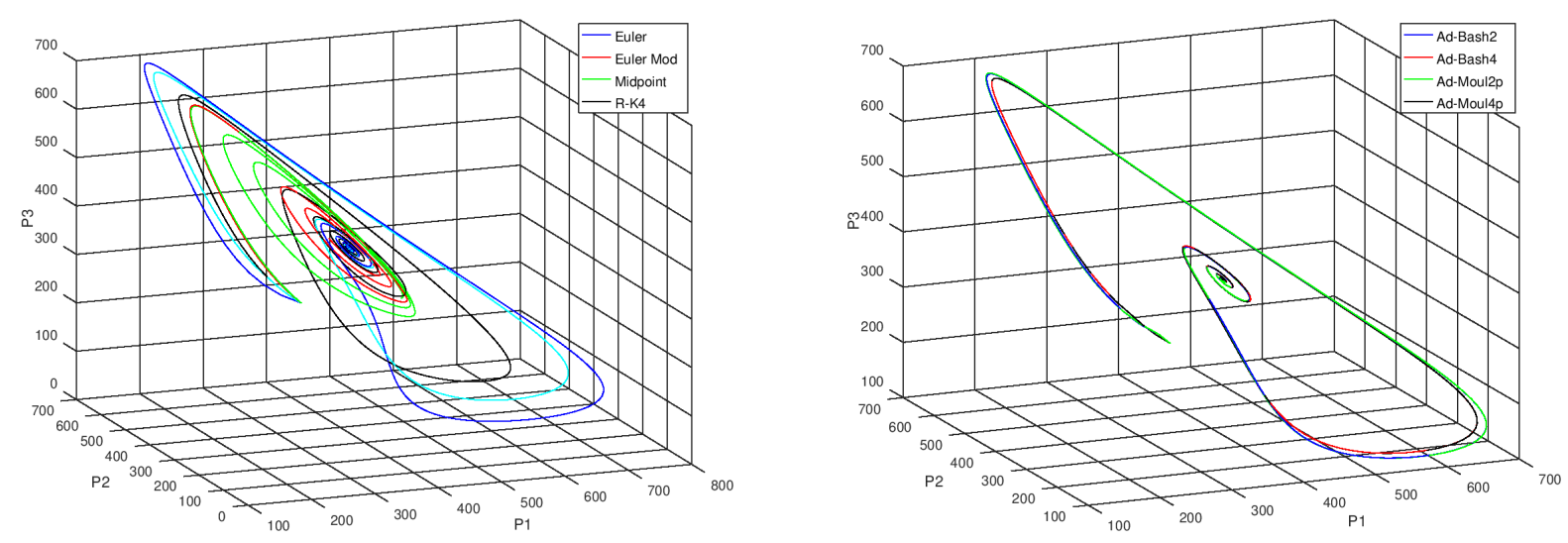

Figura 14: Espaço de fase para $P_{1}, P_{2}$ e $P_{3} \operatorname{com} h=0,25(\mathrm{~N}=800)$ e tempo final $t_{f}=20$. A curva de cor azul claro do gráfico à esquerda equivale a trajetória obtida pelo Método Ad-Moult4p, que também está no gráfico à direita.

Diminuindo o tamanho do espaçamento $h$, simulações foram implementadas para $N=3200$, isto é, $h=0,0625$ e as soluções aproximadas encontradas para $P_{1}, P_{2}$ e $P_{3}$ estão nas Figuras 11 , 12 e 13 , respectivamente.

Nesta simulação, as soluções obtidas pelos métodos de passo simples estão visualmente parecidas, apesar de ainda haver uma diferença maior entre o método de Euler e os demais métodos de passo simples, ver gráficos à esquerda das Figuras 15, 16 e 17. Entretanto, o método de Euler continua o mais parecido com a curva azul claro que se refere a solução do Método Ad-Moult4p, conforme mostra a Figura 18.

A partir dos resultados das simulações implementadas e das discussões realizadas, embasadas pelos referenciais teóricos apresentados, pode-se concluir que, nem sempre um método de maior ordem de convergência conduzirá a uma solução aproximada mais próxima da solução analítica, em comparação a métodos de menores ordens de convergência. 

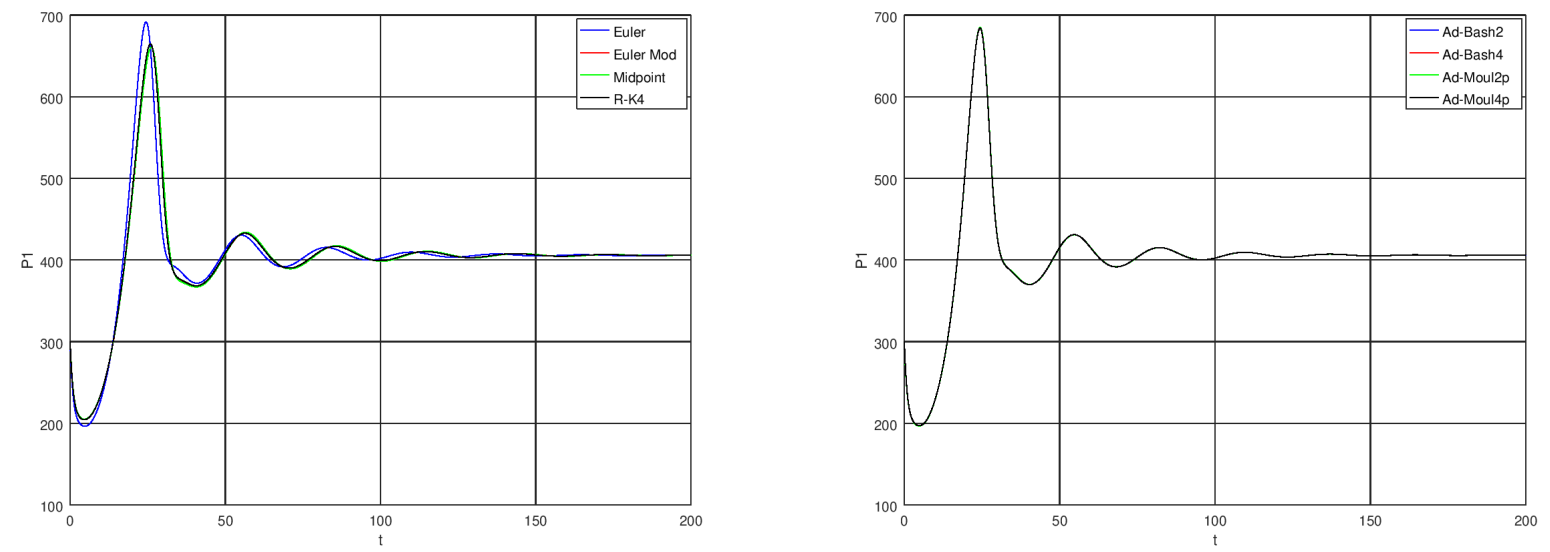

Figura 15: Comparação entre as soluções aproximadas para $h=0,0625(\mathrm{~N}=3200)$ e tempo final $t_{f}=20$ para a população $P_{1}$.
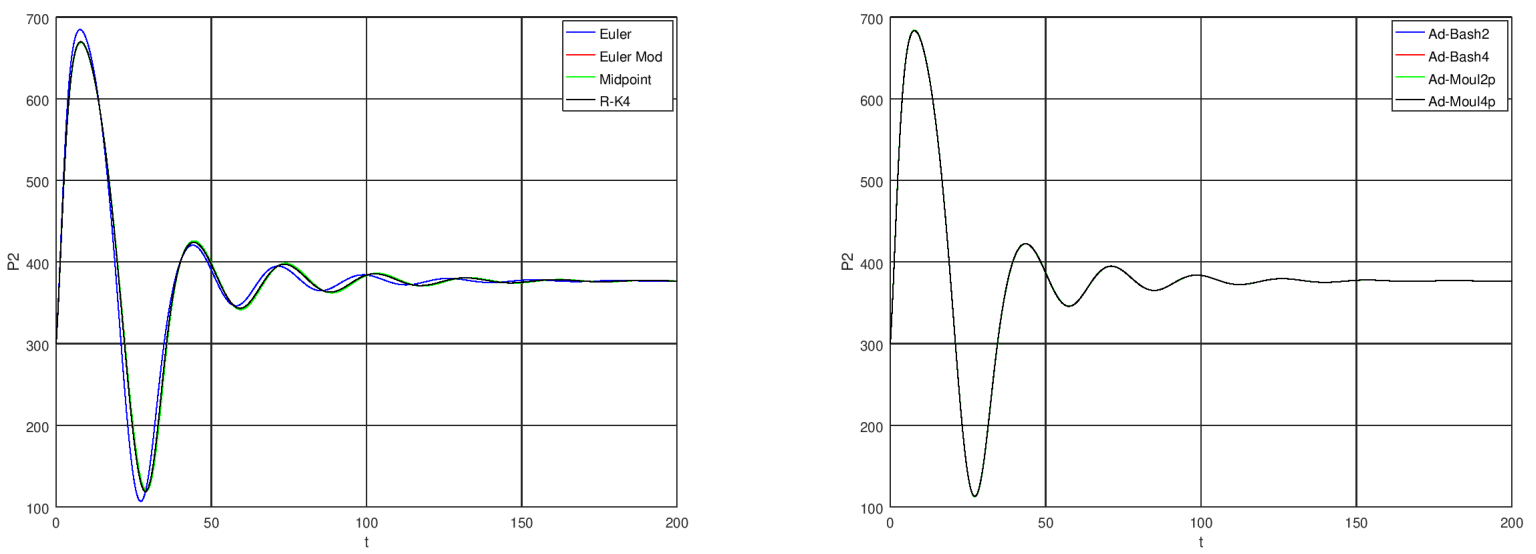

Figura 16: Comparação entre as soluções aproximadas para $h=0,0625(\mathrm{~N}=3200)$ e tempo final $t_{f}=20$ para a população $P_{2}$. 

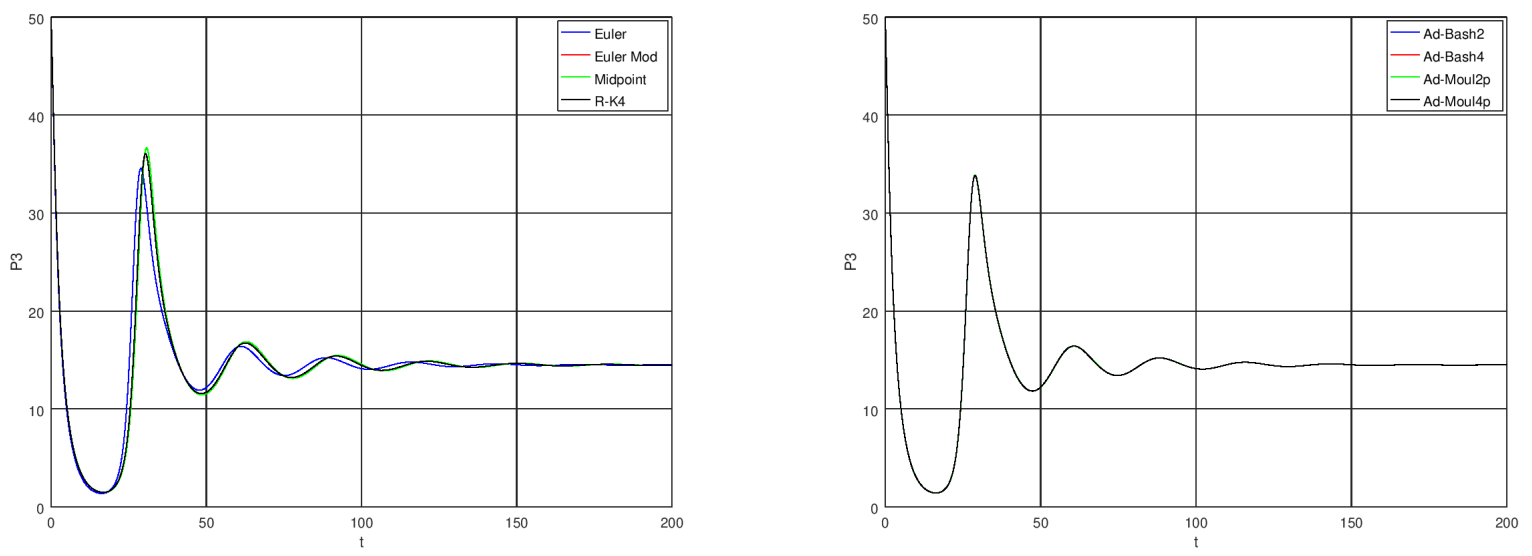

Figura 17: Comparação entre as soluções aproximadas para $h=0,0625(\mathrm{~N}=3200)$ e tempo final $t_{f}=20$ para a população $P_{3}$.
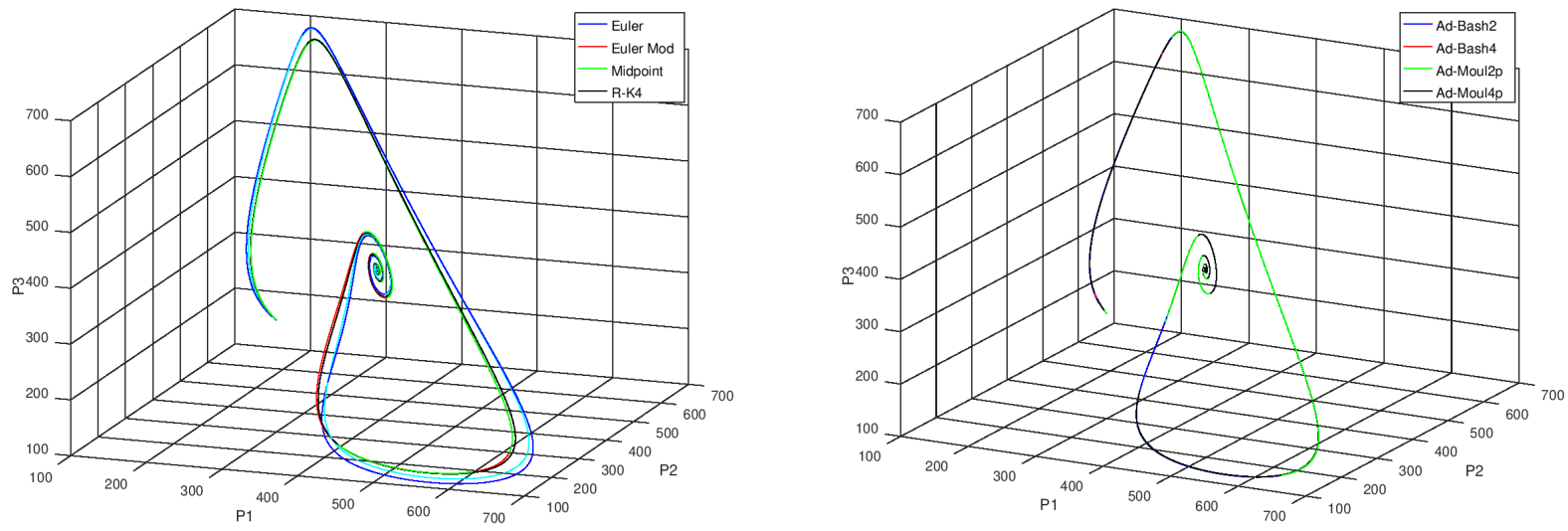

Figura 18: Espaço de fase para $P_{1}, P_{2}$ e $P_{3} \operatorname{com} h=0,0625(\mathrm{~N}=3200)$ e tempo final $t_{f}=20$. A curva de cor azul claro do gráfico à esquerda equivale a trajetória obtida pelo Método AdMoult4p, que também está no gráfico à direita. 


\section{Conclusões}

O propósito deste estudo foi realizar comparações entre métodos numéricos de equações diferenciais ordinárias, aplicadas em problemas de dinâmica populacional. Os métodos utilizados foram Euler, Euler Modificado, Ponto Médio (Midpoint), Runge-Kutta Clássico, Adams-Bashforth de 2 pontos e de 4 pontos e Adams-Moulton de 2 e 4 pontos, com estratégia de predição e correção. Um estudo numérico da ordem de convergência foi realizado considerando o erro absoluto $\left(E_{A}\right)$ e o erro relativo $\left(E_{R}\right)$, ambos comparados com a solução exata da Equação Logística da Seção 3.1. Neste exemplo foi possível observar que a medida que o espaçamento $h$ diminui, por meio do aumento da quantidade de subintervalos $N$, as soluções numéricas convergem para a solução exata em diferentes ordens, conforme ilustrado na Figura 14. Ademais, na Equação Logística foi perceptível a lenta convergência do método de Euler, com relação aos demais métodos que possuem ordens de convergência maiores.

Ao considerar o modelo de Lotka-Volterra para a dinâmica presa-predador, da Seção 3.2, percebeu-se a dificuldade dos métodos explícitos, de passo simples, em conseguir representar a solução analítica, principalmente pelo fato de que era esperado que a solução numérica mantivesse o pico das populações em um mesmo patamar, a cada ciclo completado. Entretanto, as soluções numéricas aumentaram o pico de cada população, a cada volta do ciclo representado no plano de fase. Em contrapartida, os métodos de múltiplos passos conseguiram manter os picos aproximadamente constantes, dentro do domínio computacional que implicava em torno de três ciclos.

Além disso, deve-se destacar o comportamento do método de Euler, que mesmo possuindo a menor ordem de convergência entre os esquemas explícitos de passo simples, foi o método que mais se aproximou das soluções obtidas pelos métodos implícitos de múltiplos passos.

A investigação dos resultados inesperados que ocorreram neste exemplo, ao se utilizar métodos explícitos de passo simples, conduziu a conclusão de que o modelo de Lotka-Volterra, com os parâmetros adotados nas simulações, é um problema rígido ou (stiff problem). Neste tipo de problema, nem sempre métodos explícitos de maior ordem de convergência irão fornecer soluções numéricas mais próximas das soluções analíticas, quando comparados a métodos de menor ordem de convergência.

No terceiro exemplo apresentado na Seção 3.3, foi abordada a interação entre três espécies e neste cenário, todos os métodos capturaram o ponto de equilíbrio estável. Contudo, os métodos de múltiplos passos mostraram-se mais eficientes, pois com espaçamento de $h=0,25$, todos os métodos de múltiplos passos alcançaram a mesma representação visual, enquanto que os métodos de passo simples divergiram do formato da solução (Figuras 11, 16 e 17). Isso ficou evidente nos gráficos do espaço de fase apresentados na Figura 14. Neste caso, o método de Euler teve comportamento semelhante ao verificado no exemplo da Seção 3.2, por tratar-se também de um problema rígido.

De modo geral, com esse trabalho ficou claro que ao se trabalhar com métodos numéricos é sempre aconselhável investigar e comparar as soluções numéricas, obtidas a partir de diferentes métodos, para então analisar e verificar qual ou quais são os que melhor se enquadram nas hipóteses do problema modelado pelas equações diferenciais. 


\section{Referências}

[1] ALMEIDA, E. S.; MEDEIROS, A. C.; FRERY, A. C. How good are MatLab, Octave and Scilab for computational modelling? Comput. Appl. Math., v. 31, n. 3, p. 523-538, 2012.

[2] BREWSTER, M. W. Study of free alternative numerical computational packages. SIAM, Philadelphia, v. 5, 2012.

[3] GARCIA, R. O.; SILVEIRA, G. P. Métodos numéricos aplicados às Equações de Euler: comparação entre MatLab, Octave e Fortran. Revista Eletrônica Paulista de Matemática, v. 11, p. 65-88, 2017. Disponível em: $<$ http://www.fc.unesp.br/Home/Departamentos/Matematica/revistacqd2228/v11a04metodos-numericos-aplicados-as-equacoes-de-euler.pdf>. Acesso em: 11 nov. 2018.

[4] SHARMA, N.; GOBBERT, M. K. A comparative evaluation of MATLAB, Octave and SciLab for research and teaching. HPCF Publications. 2010. Disponível em: $<$ https://userpages.umbc.edu/ gobbert/papers/SharmaGobbertTR2010.pdf >. Acesso em: 26 ago. 2018.

[5] WOUWER, A. V.; SAUCEZ, P.; VILAS, C. Simulation of ODE/PDE models with MATLAB, OCTAVE and SCILAB. Cham: Springer, 2014.

[6] BUCHANAN, J. L.; TURNER, P. R. Numerical methods and analysis. Singapore: MacGraw Hill, 1992.

[7] QUARTERONI, A.; SALERI F. Cálculo científico com MATLAB e Octave. Mailand: Springer-Verlag, 2017.

[8] BURDEN, R. L.; FAIRES, J. D.; BURDEN, A. M. Análise numérica. 3. ed. São Paulo: Cengage Learning, 2016.

[9] OCTAVE. [S.1.: s.n.], c1998-2017. Disponível em: <http://www.octave.org>. Acesso em: 26 ago. 2018.

[10] EDELSTEIN-KESHET, L. Mathematical models in biology. New York: McGraw Hill, 1988.

[11] BOYCE, W. E.; DIPRIMA, R. C. Equações diferenciais elementares e problemas de valores de contorno. 10. ed. Rio de Janeiro: LTC, 2015.

[12] SOTOMAYOR, J. Lições de equações diferenciais ordinárias. Rio de Janeiro: IMPA, 1979.

[13] ÖRNHAG, M. V. Classification of stiffness and oscillations in initial value problems. 2015. 82 f. Master's Thesis (Numerical Analysis) - Lund University, Lund, 2015.

[14] KOT, M. Elements of mathematical ecology. Cambridge: Cambridge University Press, 2001.

[15] GILPIN, M. E. Spiral chaos in a predator-prey model. The American Naturalist, v. 113, n. 2, p. 306-308, 1979. 